\title{
Estudo radiográfico digital indireto do efeito da expansão de maxila cirurgicamente assistida (EMCA) sobre o septo nasal
}

\author{
Eduardo Sanches Gonçales*, Diogo Souza Ferreira Rubim de Assis**, Ana Lúcia Álvares Capelozza***, \\ Luiz Casati Alvares****
}

\begin{abstract}
Resumo
Introdução: a deficiência transversal da maxila é caracterizada pela atresia maxilar, palato ogival, apinhamento e rotação dos dentes, além da mordida cruzada posterior (uni ou bilateral). O tratamento dos pacientes com esta deficiência consiste no alargamento das bases ósseas maxilares. Nos pacientes com maturidade esquelética, a expansão da maxila é obtida através de procedimentos ortocirúrgicos (conhecidos como expansão de maxila cirurgicamente assistida - EMCA) que podem gerar efeitos sobre a cavidade e sobre o septo nasal. Objetivo: avaliar o comportamento do septo nasal frente aos procedimentos de EMCA. Metodologia: foram avaliadas radiografias cefalométricas em norma póstero-anterior e radiografias oclusais totais de maxila, obtidas pela técnica convencional e posteriormente digitalizadas, de 16 pacientes submetidos à EMCA pela técnica de osteotomia Le Fort I subtotal. As radiografias foram obtidas no período pré-operatório (inicial) e pós-operatório mediato ou ao final da expansão e foram digitalizadas e mensuradas utilizando o programa DIGORA. Foram medidas, ainda, as distâncias entre os dentes (caninos, primeiro pré-molares, segundo pré-molares, primeiros molares e segundos molares). Resultados: após a análise estatística pode-se constatar o aumento das distâncias interdentárias e intermaxilares, além do aumento da largura da porção basal da abertura piriforme e das distâncias entre a parede lateral da porção basal da abertura piriforme e o septo nasal. Conclusão: a EMCA é um procedimento eficaz, capaz de alargar a porção basal da abertura piriforme, que parece não influenciar no posicionamento do septo nasal.
\end{abstract}

Palavras-chave: Deficiência transversal. Expansão maxilar. Septo nasal.

\footnotetext{
* Professor Assistente das Disciplinas da área de Cirurgia e Traumatologia Buco-maxilo-facial da Universidade do Sagrado Coração, Bauru/SP. Mestre em Cirurgia e Traumatologia Buco-maxilo-facial pela PUCRS. Doutor em Estomatologia da FOB - USP.

** Mestrando em Cirurgia e Traumatologia Buco-maxilo-facial da Universidade do Sagrado Coração, Bauru/SP.

*** Professora associada do Departamento de Estomatologia da FOB - USP.

**** Professor Doutor Chefe do Departamento de Radiologia da Universidade do Sagrado Coração, Bauru/SP.
} 


\section{INTRODUÇÃO}

As deformidades dentofaciais podem ser caracterizadas pelo hipo ou hiperdesenvolvimento dos ossos da face, especialmente da maxila e mandíbula, podendo essas alterações ocorrer nos sentidos transversal, vertical e ântero-posterior. A deficiência transversal de maxila caracteriza-se por maxilas atrésicas, palato ogival, apinhamento e rotação dentária, além de mordida cruzada posterior (uni ou bilateral) $)^{23}$

O tratamento dos pacientes com deficiência transversal de maxila visa aumentar as dimensões transversais, sendo que, nos indivíduos com maturidade esquelética, a expansão de maxila cirurgicamente assistida (EMCA) tem mostrado bons resultados ${ }^{19,27}$.

Os efeitos da EMCA não são observados somente no arco dentário superior; seus efeitos sobre a cavidade nasal são conhecidos desde $1961^{15}$. Alguns estudos relataram melhora na respiração nasal decorrente do aumento do volume nasal e do diâmetro transversal da maxila ${ }^{3,12,16}$.

O aumento ocorre pelo movimento lateral das paredes laterais da cavidade nasal, à medida que as estruturas maxilares separam-se, resultando em aumento do espaço interno nasal ${ }^{4}$, uma vez que a maxila participa na composição da parede lateral nasal24.

O septo nasal osteocartilaginoso divide a cavidade nasal em duas, sendo que seu esqueleto, em sua porção óssea, está constituído pela lâmina perpendicular do osso etmóide, súpero-anteriormente e pelo vômer, súpero-posterior e inferiormente, articulado diretamente às maxilas através de suas cristas nasais $^{19}$.

A EMCA requer a utilização de técnica cirúrgica. Inúmeras técnicas cirúrgicas e variações têm sido descritas ${ }^{5,6,7,9,13,15,20,25,26,30}$, porém, mesmos as técnicas mais invasivas ${ }^{9,30}$ não realizam osteotomia total do septo nasal e são essenciais no tratamento de pacientes com idade mais avançada ${ }^{2}$.

Em 1985, não foi encontrada mudança na posição do septo, mas aumento significativo do espaço aéreo em imagens tomográficas coronais utilizadas para avaliação dos efeitos da expansão maxilar na anatomia da cavidade nasal ${ }^{28}$.

Em 1994, houve a padronização da radiografia cefalométrica em norma póstero-anterior, o que permitiu a reprodução da técnica ${ }^{8}$. Em 1995, o cefalograma póstero-anterior foi considerado o melhor método de identificação e avaliação das discrepâncias transversais esqueléticas, pois permite a superposição das radiografias e a comparação de medidas lineares ${ }^{9}$.

Por quase um século, o filme radiográfico convencional registrou a imagem radiográfica bidimensional. Atualmente uma imagem obtida mais rapidamente e com diminuição de exposição ao paciente pode ser obtida com técnicas digitais ${ }^{10,11,18}$.

Dentre as possibilidades de utilização das imagens radiográficas digitais destaca-se a execução de tarefas específicas, como mensuração precisa, maior escala de contraste, decorrente da maior variedade de tons da escala de cinza ${ }^{21,22,29}$. Considerando essas características, o sistema Digora mostrou melhores condições de trabalho, quando comparado com outros sistemas ${ }^{10}$.

O objetivo deste trabalho foi avaliar, através de mensurações realizadas em telerradiografias cefalométricas em norma póstero-anterior e digitalizadas de pacientes submetidos à EMCA, o comportamento do septo nasal.

\section{MATERIAL E MÉTODOS}

Avaliou-se 32 telerradiografias cefalométricas em norma póstero-anterior e 32 radiografias oclusais totais de maxila, padronizadas ${ }^{1}$, de 16 pacientes adultos atendidos no Ambulatório de $\mathrm{Ci}$ rurgia Ortognática da Clínica de Odontologia da Universidade do Sagrado Coração - USC, Bauru/ SP, portadores de deficiência transversal maxilar, podendo tal anomalia estar associada a outras más formações.

Todos os indivíduos considerados neste estudo submeteram-se à EMCA, utilizando-se modificação da técnica cirúrgica descrita por Betts et al. ${ }^{9}$ 
e operados pela mesma equipe cirúrgica (tal modificação consistiu em não realizar osteotomia na porção ântero-inferior do septo nasal); as radiografias utilizadas neste estudo foram obtidas nos períodos pré-operatório (inicial) e pós-operatório mediato ou final (2 meses).

As telerradiografias cefalométricas em norma póstero-anterior foram obtidas em aparelho radiográfico Rotograph 230 eur (marca Dabi - atlante), com regime de trabalho de $85 \mathrm{Kv}$ e 3 segundos de exposição, o filme utilizado para o registro das imagens foi o Kodak Mxg/Plus e ecran Kodak Lanex Regular, processados em processadora Macrotec MX - 2. As radiografias intrabucais oclusais totais de maxila foram obtidas em um aparelho Dabi-Atlante, com filme Kodak Insight e 0,8s de exposição, obtidas e processadas de maneira padronizada.

Todos os pacientes foram submetidos à avaliação por profissionais da área de Ortodontia e Cirurgia e Traumatologia Bucomaxilofacial, para diagnóstico e plano de tratamento. Utilizou-se para o diagnóstico da deficiência transversal de maxila: anamnese, exame clínico, modelos de estudo padronizados, fotografias intrabucais e da face em normas frontal e lateral, radiografias panorâmicas, oclusais totais de maxila e telerradiografias cefalométricas em norma lateral e póstero-anterior.

Com a atresia maxilar diagnosticada, os indivíduos foram encaminhados para avaliação e acompanhamento ortodôntico para instalação de dispositivo de expansão (aparelho disjuntor palatino dento-suportado), o que permitiu a realização do procedimento cirúrgico segundo o seguinte protocolo:

- procedimento realizado em âmbito hospitalar, sob anestesia geral endovenosa total;

- técnica cirúrgica com exposição maxilar bilateral e osteotomia tipo Le Fort I sub-total, com liberação dos pilares nasais, zigomáticos e pterigoideos, conforme descrito por Betts et al. ${ }^{9}$;

- sem ativação imediata e período de repouso de 5 (cinco) dias até o início da expansão;
- ativação inicial de $1 \mathrm{~mm}$ no quinto dia;

- ativações contínuas a partir do sexto dia, de $0,5 \mathrm{~mm}$ por dia, durante 10 dias, com interrupção por mais 7 dias para reavaliação e posterior continuidade da expansão (se necessário);

- o ciclo citado acima se repetiu até que as vertentes triturantes das cúspides palatinas dos molares e pré-molares superiores estivessem em contato com as vertentes triturantes das cúspides vestibulares dos dentes inferiores posteriores;

- período de contenção mínimo de 4 meses, sendo sua indicação baseada na interpretação radiográfica;

- liberação para continuidade do tratamento ortodôntico.

As telerradiografias cefalométricas em norma póstero-anterior e oclusais totais de maxila, obtidas nos períodos supracitados, foram digitalizadas em scanner de mesa UMAX Astra 2400s, no tamanho original e com resolução de 600 dpi e arquivadas em formatoTIFF no Sistema Digora.

Nas telerradiografias cefalométricas em norma póstero-anterior foram feitas as seguintes medidas: largura da base da abertura piriforme (LBAP), largura média da abertura piriforme (LMAP), largura superior da abertura piriforme (LSAP), base da parede lateral da abertura piriforme ao septo do lado direito (BAPSD) (Fig. 1), base da parede lateral da abertura piriforme ao septo do lado esquerdo (BAPSE), largura média da parede lateral da abertura piriforme ao septo do lado direito (LMAPSD), largura média da parede lateral da abertura piriforme ao septo do lado esquerdo (LMAPSE), largura superior da parede lateral da abertura piriforme ao septo do lado direito (LSAPSD) e largura superior da parede lateral da abertura piriforme ao septo do lado esquerdo (LSAPSE) (Fig. 2). Nas radiografias oclusais totais de maxila, mensurou-se a distância intermaxilar crista óssea cervical (DIMCOC), distância intermaxilar média (DIMM) e distância intermaxilar posterior (DIMP) (Fig. 3); todas as mensurações foram realizadas no Sistema Digora, por meio de 


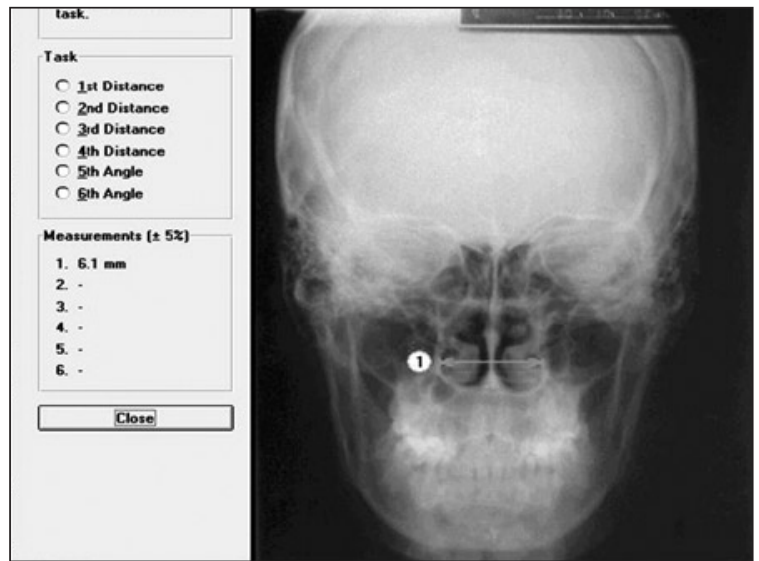

FIGURA 1 - Representação da utilização do Sistema DIGORA - medida da base da parede lateral da abertura piriforme ao septo do lado direito e esquerdo.

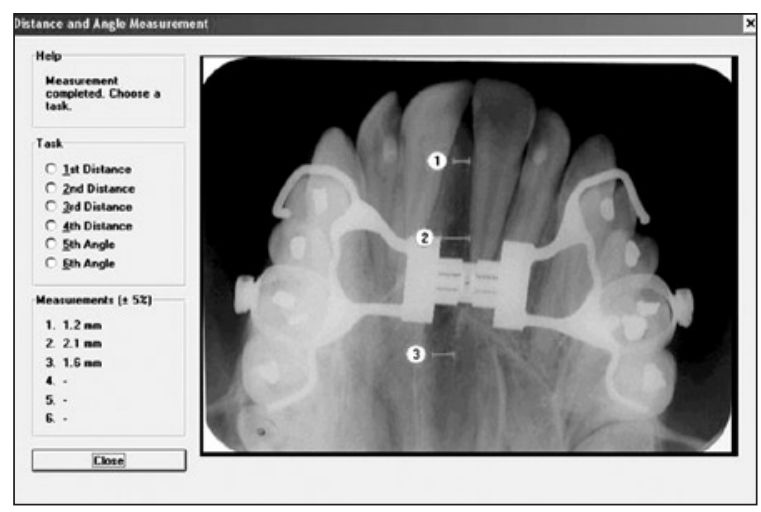

FIGURA 3 - Representação da utilização do Sistema DIGORA - onde se visualiza a confecção das medidas da distância intermaxilar.

ferramenta específica para esse fim.

Obteve-se ainda a distância intercaninos (DC), distância entre ${ }^{\text {os }}$ pré-molares (D1PM), distância entre $2^{\text {os }}$ pré-molares (D2PM), distância entre $1^{\text {os }}$ molares $(\mathrm{D} 1 \mathrm{M})$ e distância entre $2^{\text {os }}$ molares (D2M), utilizando-se um compasso de pontas secas, diretamente nos dentes dos pacientes, em períodos pré-operatório (inicial) e pós-operatório de 2 meses (final), avaliando a expansão clínica obtida.

Após a coleta e tabulação dos dados, procedeuse a análise estatística dos mesmos; utilizando-se

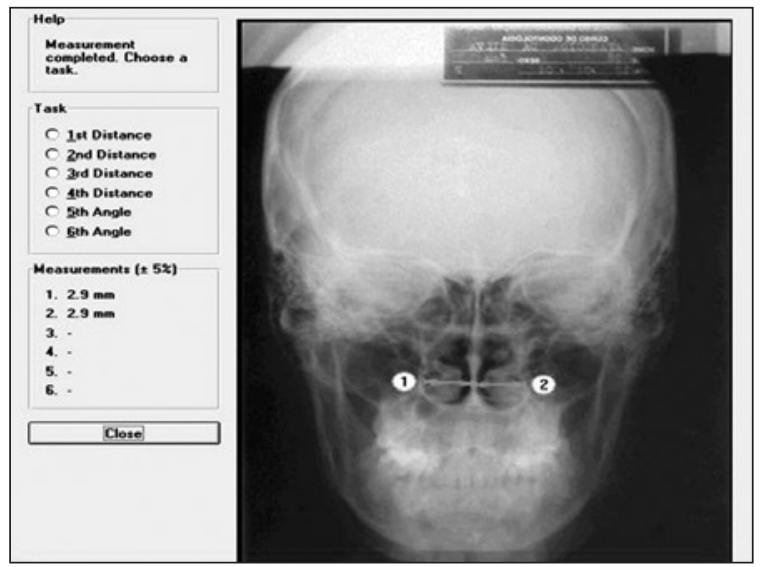

FIGURA 2 - Representação da utilização do Sistema DIGORA - medida da largura da base da abertura piriforme.

o teste $t$ de Student para amostras pareadas (para os dados obtidos a partir das mensurações nas telerradiografias cefalométricas póstero-anteriores e nas distâncias interdentárias), nas medidas obtidas a partir das radiografias oclusais totais de maxila utilizou-se o teste não paramétrico de Wilcoxon, caracterizando o comportamento do septo nasal dos pacientes submetidos à EMCA.

\section{RESULTADOS}

$\mathrm{Na}$ amostra de 16 indivíduos (13 mulheres e 3 homens) a idade média foi de 26,5 anos (variação entre 18 e 38 anos). Os resultados obtidos revelaram aumento nas distâncias interdentárias, na largura da base da abertura piriforme (LBAP) e nas distâncias da base da abertura piriforme ao septo nasais (BAPSD e BAPSE) estatisticamente significantes, enquanto a largura média (LMAP) e superior (LSAP) da abertura piriforme e as distâncias da parede lateral direita e esquerda da abertura piriforme média e superior ao septo não mostraram variação estatisticamente significante (Tab. 1). As mensurações realizadas nas radiografias oclusais totais de maxila, na porção superior (DIMCOC), média (DIMM) e posterior (DIMP) mostraram aumento estatisticamente significantes (Tab. 2). 
Tabela 1 - Variável, média $(\mathrm{mm})$ e desvio-padrão $(\mathrm{mm})$ das medidas iniciais e finais, diferença entre inicial e final (mm) e resultado do teste estatístico de 16 pacientes submetidos à EMCA.

\begin{tabular}{|c|c|c|c|c|}
\hline variável & $\begin{array}{c}\text { medidas iniciais } \\
\text { (média } \pm \text { desvio-padrão) }\end{array}$ & $\begin{array}{c}\text { medidas finais } \\
\text { (média } \pm \text { desvio-padrão) }\end{array}$ & $\begin{array}{c}\text { diferença entre } \\
\text { inicial e final }\end{array}$ & $\begin{array}{c}\text { resultado do teste } \\
\text { estatístico }\end{array}$ \\
\hline$D C$ & $27,43 \pm 2,94$ & $34,29 \pm 3,30$ & 6,86 & $p<0,001$ \\
\hline D1PM & $28,56 \pm 3,88$ & $36,25 \pm 3,30$ & 7,69 & $p<0,001$ \\
\hline $\mathrm{D} 2 \mathrm{PM}$ & $33,60 \pm 5,39$ & $41,73 \pm 3,64$ & 8,13 & $p<0,001$ \\
\hline D1M & $39,81 \pm 5,50$ & $47,23 \pm 4,19$ & 7,42 & $p<0,001$ \\
\hline $\mathrm{D} 2 \mathrm{M}$ & $46,92 \pm 4,44$ & $54,08 \pm 3,50$ & 7,88 & $p<0,001$ \\
\hline LBAP & $4,86 \pm 0,49$ & $5,29 \pm 0,49$ & 0,43 & $p<0,05$ \\
\hline LMAP & $3,55 \pm 0,50$ & $3,66 \pm 0,58$ & 0,11 & $p>0,05$ \\
\hline LSAP & $1,68 \pm 0,29$ & $1,72 \pm 0,30$ & 0,04 & $p>0,05$ \\
\hline BAPSD & $2,31 \pm 0,35$ & $2,59 \pm 0,26$ & 0,28 & $p<0,05$ \\
\hline BAPSE & $2,20 \pm 0,34$ & $2,52 \pm 0,35$ & 0,32 & $p<0,05$ \\
\hline LMAPSD & $1,71 \pm 0,40$ & $1,74 \pm 0,43$ & 0,03 & $p>0,05$ \\
\hline LMAPSE & $1,57 \pm 0,32$ & $1,68 \pm 0,42$ & 0,11 & $p>0,05$ \\
\hline LSAPSD & $0,79 \pm 0,76$ & $0,80 \pm 0,17$ & 0,01 & $p>0,05$ \\
\hline LSAPSE & $0,71 \pm 0,15$ & $0,79 \pm 0,21$ & 0,08 & $p>0,05$ \\
\hline
\end{tabular}

Tabela 2 - Variável, mediana e semi-amplitude total das medidas inicial e final (mm), diferença entre final e inicial (mm) e resultado do teste estatístico (WILCOXON) de 16 pacientes submetidos à EMCA.

\begin{tabular}{ccccc}
\hline variável & $\begin{array}{c}\text { medidas iniciais (mediana } \\
\pm \text { semi-amplitude total) }\end{array}$ & $\begin{array}{c}\text { medidas finais (mediana } \\
\pm \text { semi-amplitude total) }\end{array}$ & $\begin{array}{c}\text { diferença entre } \\
\text { final e inicial }\end{array}$ & $\begin{array}{c}\text { resultado do } \\
\text { teste estatístico }\end{array}$ \\
\hline DIMCOC & $0,25 \pm 0,20$ & $3,20 \pm 1,80$ & 2,95 & 2,3 \\
DIMM & $0,10 \pm 0,15$ & $2,40 \pm 1,45$ & 1,45 & $\mathrm{p}<0,001$ \\
DIMP & $0,00 \pm 0,10$ & $1,45 \pm 1,05$ & $\mathrm{p}<0,001$ \\
\hline
\end{tabular}

\section{DISCUSSÃO}

A deficiência transversal de maxila é caracterizada por atresia maxilar, palato ogival, apinhamento e rotação dentária e mordida cruzada posterior uni ou bilateral ${ }^{23}$. O tratamento dos pacientes portadores desta deficiência tem como objetivo aumentar a dimensão transversal da maxila. Nos indivíduos com maturidade esquelética, a expansão de maxila cirurgicamente assistida (EMCA) tem mostrado bons resultados ${ }^{19,27}$ e seus efeitos podem ser observados também na cavidade nasal ${ }^{3,15,16}$, podendo melhorar a respiração dos pacientes com o aumento do volume nasal ${ }^{12}$.

O aumento do volume da cavidade nasal ocorre pelo movimento lateral das paredes laterais da cavidade nasal, com a separação das estruturas maxilares, resultando em aumento do espaço nasal interno ${ }^{4}$.
Apesar de alguns autores ${ }^{28}$ não terem encontrado mudanças na posição do septo nasal, a descrição anatômica desta estrutura revelou que ela pode interferir na expansão da maxila ou movimentar-se conjuntamente durante a expansão ${ }^{17}$.

Dentre as inúmeras técnicas cirúrgicas descritas na literatura para expansão de maxila $a^{5,6,7,9,13,}$ $15,20,25,26,30$, destacam-se as mais invasivas ${ }^{9,30}$, como essenciais no tratamento de pacientes com idade mais avançada ${ }^{2}$, porém essas técnicas não preconizam a realização de osteotomia total do septo nasal, favorecendo assim sua interferência com a expansão ou a possibilidade de movimento, que pode acarretar desvio.

Em nosso trabalho, os resultados mostram que a EMCA acarreta alterações na cavidade nasal, que são traduzidas por discreto aumento de volume, decorrente do alargamento das bases apicais 
maxilares, observando-se aumento da largura total da abertura piriforme apenas em sua porção basal. Da mesma forma, observou-se ainda que o septo nasal parece não alterar sua posição, pois as distâncias do septo à parede lateral nasal direita e esquerda mantiveram-se proporcionais.

Destaca-se também, neste estudo, o fato de as distâncias interdentárias terem aumentado cerca de $7,5 \mathrm{~mm}$ (em média) e as distâncias intermaxilares, verificadas na região da sutura intermaxilar, também aumentaram, confirmando que o procedimento de EMCA foi efetivo, não ocorrendo a correção da mordida cruzada apenas por movimento dentário, e sim por deslocamento lateral das maxilas.

Importante salientar que o método utilizado permite a utilização de radiografias que podem ser padronizadas e reproduzidas ${ }^{8}$ e que oferecem boa visualização da fossa nasal ${ }^{14}$, contudo pode-se observar melhor qualidade de imagem quando utili- zado o sistema de radiografia digital e os recursos de aferição que o sistema Digora oferece ${ }^{10}$. Nesse estudo, optou-se pela utilização do sistema Digora para as aferições e por sua disponibilidade no Departamento de Estomatologia da FOB-USP.

\section{CONCLUSÃO}

Em face aos resultados obtidos e no exposto acima, pode-se concluir que:

a) A EMCA é um procedimento eficaz;

b) A EMCA possui discreto efeito nas dimensões da cavidade nasal e no posicionamento do septo nasal;

c) O efeito mais observado da EMCA sobre a cavidade nasal é o alargamento da base da cavidade nasal.

\title{
Indirect digital radiographic study about the effect of surgically assisted maxillary expansion (SAME) on the nasal septum
}

\begin{abstract}
Introduction: Maxillary transversal deficiency is characterized by constricted maxillary arch, dental crowding and rotation, along with posterior uni or bilateral crossbite. The treatment of this deficiency is based on the widening of maxillary bone. In skeletal mature patients the maxillary expansion is achieved by means of surgical-orthodontic procedures (known as surgically assisted maxillary expansion - SAME) that can cause some effects on the nasal cavity and septum. Aim: To evaluate the behavior of the nasal septum after the procedures of SAME. Methods: Post-anterior cephalometric and maxillary occlusal radiographs, taken by conventional technique and therefore digitalized, were analyzed from 16 patients undergone SAME. For all patients, the SAME technique consisted of subtotal Le Fort I osteotomy. The radiographs were taken before the surgical procedure, right after (mediate post-operatory time) or after the expansion, and thereafter digitalized and measured using DIGORA program. Also, the distances between teeth (canines, first premolars, second premolars, first molars and second molars) were registered. Results: The statistical treatment showed an increase in interdental and intermaxillary distances, along with an increase in the width of the base of piriform aperture and in the distances between the base of lateral wall of the piriform aperture and nasal septum. Conclusion: SAME is an efficient procedure that seems not to influence the septum position.
\end{abstract}

Key words: Maxillary transversal deficit. Maxillary expansion. Nasal septum. 


\section{REFERÊNCIAS}

1. AlVARES, L. C.; TAVANO, O. Curso de Radiologia em Odontologia. São Paulo: Ed. Santos, 1998. p. 248.

2. ANTILLA, A. et al. Feasibility and long term stability of surgically assisted rapid palatal expansion with lateral osteotomy. Eur. J. Orthod., Oxford, v. 26, p. 391-395, 2004.

3. BABACANA, H. et al. Rapid maxillary expansion and surgically assisted rapid maxillary expansion effects on nasal. Angle Orthod., Appleton, v. 76, p. 66-71, 2006.

4. BASCIFTCI, F. A. et al. Does the timing and method of rapid maxillary expansion have an effect on the changes in nasal dimensions? Angle Orthod., Appleton, v. 72, p. 118-123, 2002.

5. BASDRA, E. K.; ZOLLER, J. E.; KOMPOSH, G. Surgically assisted rapid palatal expansion. J. Clin. Orthod., Boulder, v. 29, no. 12, p. 762-766, Dec. 1995.

6. BELL, W. H.; EPKER, B. N. Surgical-orthodontic expansion of the maxilla. Am. J. Orthod., St. Louis, v. 70, no. 5, p. 517-528, 1976.

7. BELL, W.; JACOBS, J. D. Surgical - orthodontic correction of horizontal maxillary deficiency. J. Oral Surg., Chicago, v. 37, p. 987-902,1979.

8. BETTS, N. J.; LISEMBY, W. C. Normal adult transverse jaw values obtained using standardized poster anterior cephalometrics. J. Dent. Res., Alexandria, v. 73, p. 298, 1994. IADR Abstracts.

9. BETTS, N. J et al. Diagnosis and treatment of transverse maxillary deficiency. Int. J. Adult Orthod. Orthognath. Surg., Chicago, v. 10, no. 2, p. 75-96, 1995.

10. BÓSCOLO, F. N. et al. Clinical study of the sensitivity and dynamic range of three digital systems, e-speed film and digitized film. Braz. Dent. J., Ribeirão Preto, v. 12, no. 3, p. 191-195, 2001.

11. BROCKLEBANK, L. Dental radiology: understanding the X-ray Image. Oxford: Oxford University Press, 1997. p. 217.

12. COMPADRETTI, G. C. et al. Acoustic rhinometric measurements in children undergoing rapid maxillary expansion. Int. J. Pediatr. Otorhinolaryngol., Amsterdam, v. 70, p. 27-34, 2006.

13. GLASSMAN, A. S. et al. Conservative surgical orthodontic adult rapid palatal expansion: sixteen cases. Am. J. Orthod., St. Louis, v. 86, no. 3, p. 207-213, 1984.

14. GOAZ, P. W.; WHITE, S. C. Oral radiology: principles and interpretation. 3rd ed. St. Louis: Mosby, 1994.

15. HASS, A. J. Rapid expansion of the maxillary dental arch and nasal cavity by opening the midpalatal suture. Angle Orthod. Appleton, v. 31, no. 2, p. 73-90, 1961.

16. KOUDSTAAL, M. J.; POORT, L. J.; VAN DER WAL, K. G. H.; WOLVIUS, H. B.; PRAHL-ANDERSEN, B.; SCHULTEN, A. M. J. Surgically assisted rapid maxillary expansion (sarme): a review of the literature. Int. J. Oral Maxillofac. Surg., Copenhagen, v. 34, p. 709-714, 2005.

17. LANG, J. Clinical anatomy of the nose, nasal cavity and paranasal sinuses. 1st ed. New York: Thieme Medical, 1989. p. 144.

18. LANGLAND, O.; LANGLAIS, B. Princípios de diagnóstico por imagem em Odontologia. São Paulo: Ed. Santos, 2002.

19. LANIGAN, D. T.; MINTZ, S. M. Complications of surgically assisted rapid palatal expansion: review of the literature and report of a case. J. Oral Maxillofac. Surg., Philadelphia, v. 60, p. 104-110, 2002.

20. LEHMAN, J. A.; HASS, A. J.; HASS, D. G. Surgical orthodontic correction of transverse maxillary deficiency: a simplified approach. Plast. Reconstr. Surg., Hagerstown, v. 73, no. 1, p. 62-68, 1984.

21. MAURIELLO, S. M.; PLATIN, E. Dental digital radiographic imaging. Int. J. Dent. Hyg., Oxford, v. 75, p. 323-331, 2001

22. MILES, D. A. The deal on digital: the status of radiographic imaging. Compend. Contin. Educ. Dent., Jamesburg, v. 22, no. 12, p. 1057-1062, Dec. 2001.

23. NARY FILHO, H.; GONÇALES, E. S.; RIBEIRO JR., P. D. Abordagem cirúrgica voltada para a estética. Biodonto, Rio de Janeiro, v. 1, n. 1, p. 2-11, mar./abr. 2004.
24. NAVARRO, J. C. M. Cavidade do nariz e seios paranasais. Bauru: All Dent, 1997.

25. NORTHWAY, W. M.; MEADE JR., J. B. Surgically assisted rapid maxillary expansion: a comparison of techniques, response and stability. Angle Orthod., Appleton, v. 67, no. 4, p. 309-320, 1997.

26. POGREL, M. A. et al. Surgically assisted rapid maxillary expansion in adults. Int. J. Adult Orthod. Orthognath. Surg., Chicago, v. 7, p. 37-41, 1992.

27. RIBEIRO JR., P. D. et al. Avaliação clínica dos procedimentos de expansão cirurgicamente assistida de maxila (ECAM). $\mathbf{R}$. Dental Press Ortodon. Ortop. Facial, Maringá v. 11, n. 1, p. 44-49, 2006.

28. SCHWARZ, G. M. et al. Tomographic assessment of nasal septal changes following surgical-orthodontic rapid maxillary expansion. Am. J. Orthod., St. Louis, v. 87, no. 1, p. 39-45, 1985.

29. SCHULZE, R. K. W.; ROSING, S. T. R.; D'HOEDT, B. Contrast perception in digitized panoramic radiographs compared with their film-based origin. Oral Surg. Oral Med. Oral Pathol. Oral Radiol. Endod., St. Louis, v. 94, p. 3388-3394, 2002.

30. WOODS, M.; WISENFELD, D.; PROBERT, T. Surgically - assisted maxillary expansion. Aust. Dent. J., Sydney, v. 42, no. 1 , p. 38-42, 1997.
Endereço para correspondência

Eduardo Sanches Gonçales

Rua Henrique Savi, 5-63

CEP: 17.011-590 - Bauru/SP

E-mail: eduardogoncales@iocp.com.br 